\title{
SÍNDROME DE BURNOUT EN MÉDICOS DE UN HOSPITAL DE LA AMAZONÍA PERUANA
}

\author{
BURNOUT SYNDROME IN PHYSICIANS OF A HOSPITAL IN THE PERUVIAN AMAZON
}

Jennifer Vilchez-Cornejo ${ }^{1,2, a}$, Luccio Romaní-Ojeda ${ }^{3, b}$, Katerine Ladera-Porta ${ }^{2, b}$, Mario Marchand-Gonzales ${ }^{1, a}$

\begin{abstract}
RESUMEN
Objetivo: Determinar el Síndrome de Burnout (SBO) en médicos cirujanos de los consultorios externos de un hospital de la Amazonía peruana. Métodos: Estudio transversal analítico; que incluyó a 30 médicos cirujanos que atendieron en los consultorios externos de las especialidades de Medicina, Cirugía, Gineco-Obstetricia y Pediatría del Hospital Regional de Pucallpa, determinando el agotamiento profesional mediante el Inventario de Burnout de Maslach (MBI) y la percepción de la relación médico paciente mediante la escala PREMEPA. Se emplearon modelos lineales generalizados para evaluar razones de prevalencia cruda y ajustada. Resultados: El 76,7\% de los médicos fueron varones, ocho de cada diez padecieron SBO. El 20\% de los pacientes estuvieron satisfechos con la consulta y sólo el $16,7 \%$ indicó tener una mediana relación médico paciente. Se encontró asociación estadísticamente significativa entre quienes padecían SBO y se atendieron en las consultas externas de los servicios de cirugía (RPa: 1.46; IC 95\%: 1.20-1.76) , gineco obstetricia (RPa: 1.69; IC 95\%: 1.41-2.03) o en aquellos pacientes que se encontraban insatisfechos con la consulta brindada (RPa: 1.59; IC 95\%: 1.22-2.07), el haber realizado el residentado médico fue un factor protector a desarrollar SBO (RPa: 0.62; IC 95\%: 0.620.77). Conclusión: Los pacientes manifiestan tener niveles adecuados de relación médico-paciente a pesar del elevado índice de SBO en médicos, las especialidades quirúrgicas predisponen a desarrollar Burnout..
\end{abstract}

Palabras clave: Agotamiento Profesional; Relaciones Médico-Paciente; Médicos. (fuente: DeCS BIREME)

\begin{abstract}
Objective: To determine Burnout Syndrome (SBO) in physicians treated in the outpatients clinic of a hospital in the Peruvian Amazon during 2017. Methods: Analytical cross-sectional study; which included 30 medical surgeons who attended in the external offices of the specialties of Internal Medicine, General Gynecology and Pediatric Surgery of the Regional Hospital of Pucallpa, professional exhaustion was determined through the Maslach Burnout Inventory (MBI) and the perception of the patient-doctor relationship using the scale (PREMEPA). Generalized linear models were performed by prevalence ratios crude and adjusted estimated with a $95 \%$ confidence interval. Results: $76.7 \%$ of the doctors were male, eight out of ten suffered Burnout. $20 \%$ of the patients were satisfied and only $16.7 \%$ indicated they had a median physician-patient medical relationship. Statistically significant association was found among those suffering from Burnout and the outpatient offices were treated of surgery services (RPa: 1.46; $95 \% \mathrm{Cl}: 1.20-1.76)$, obstetric gynecology (RPa: 1.69; $95 \% \mathrm{Cl}: 1.41-2.03$ ) or in those patients who were dissatisfied with the consultation provided (RPa: 1.59; $95 \% \mathrm{Cl}: 1.22-2.07)$, having medical residency was a protective factor to develop Burnout (RPa: $0.62 ; 95 \% \mathrm{Cl}$ : 0.62- 0.77). Conclusion: Patients claim to have adequate levels of physician-patient relationship despite the high rate of doctors suffering from Burnout, surgical specialties predispose doctors to develop Burnout. However, having performed medical residency predisposed the doctor to have less Burnout Syndrome.
\end{abstract}

Key words: Burnout; Physician-Patient Relations; Physicians. (source: MeSH NLM)

\footnotetext{
${ }^{1}$ Red Asistencial Ucayali, Hospital Essalud II Pucallpa, Ucayali-Perú.

${ }^{2}$ Facultad de Medicina Humana, Universidad Nacional de Ucayali, Ucayali-Perú

${ }^{3}$ Facultad de Medicina Humana, Universidad de San Martín de Porres, Chiclayo-Perú.

a Médico cirujano.

b Estudiante de medicina humana.
}

Citar como: Jennifer Vilchez-Cornejo, Luccio Romaní-Ojeda, Katerine Ladera-Porta, Mario Marchand-Gonzales. Síndrome de burnout en médicos de un hospital de la Amazonía peruana. Rev. Fac. Med. Hum. Octubre 2019; 19(4):60-67. DOI 10.25176/RFMH.v19i4.2220 


\section{INTRODUCCIÓN}

El síndrome de burnout (SBO), es considerado como un estado de estrés emocional y psicológico, experimentado por el personal de salud que atiende a pacientes estructuralmente vulnerables ${ }^{1}$, además, suele presentarse en ambientes heterogéneos, desde los hospitales en comunidades urbanas hasta los entornos de salud globales ${ }^{2,3}$. Diversos investigadores ${ }^{4}$ lograron describirlo como una combinación de agotamiento emocional, despersonalización y bajo rendimiento personal debido al estrés crónico de la práctica médica. Actualmente, se considera como una respuesta al estrés laboral crónico que experimentan diversos profesionales cuyo contacto directo con las personas es fundamental y es pieza clave de la labor que realizan ${ }^{5,6}$

Se ha llegado a considerar un problema de salud mental, por tratarse de un sobreesfuerzo sostenido a lo largo del tiempo que puede aumentar el riesgo a contraer enfermedades mentales e incluso cardiovasculares ${ }^{7,8}$; sin mencionar el potencial de afectar negativamente el rendimiento y la calidad del servicio durante el desempeño de las funciones laborales del profesional 9 .

A nivel mundial, se ha reportado que alrededor del $45 \%$ de médicos han tenido o tienen SBO, esto incluye a médicos generales y a médicos residentes, en quienes puede llegar hasta un $80 \%$ de casos, por ser un personal que realiza mayor actividad ${ }^{10}$. El estrés de los profesionales de la salud es considerablemente más alto respecto a otras profesiones, oscilando entre un 30 y $40 \%$ más $^{11}$, a causa de lidiar con el sufrimiento humano, largas jornadas laborales $y$, en múltiples ocasiones, un salario insuficiente al que se ven sometidos constantemente.

En el Perú, el estudio más grande realizado en el año 2014, encontró una prevalencia mayor en médicos $(3,7 \%)$ que en enfermeras $(2,1 \%)^{12}$. Por todo lo mencionado, se podría considerar que los médicos son un grupo vulnerable a experimentar SBO, lo cual podría predisponerlos a brindar una mala calidad en la atención a los pacientes y el aumento de los errores médicos; sin mencionar el detrimento de la salud al que son expuestos ${ }^{13}$.

La presente investigación surge de la necesidad de determinar los factores asociados al Síndrome de Burnout en médicos cirujanos de un hospital de la Amazonía peruana; así como su repercusión en la relación médico paciente.

\section{MÉTODOS}

Estudio transversal analítico realizado durante octubre del 2017 dirigido a los médicos cirujanos que atendieron en los consultorios externos de los servicios de medicina, cirugía, gineco obstetricia y pediatría del Hospital Regional de Pucallpa.

Se incluyeron a aquellos médicos cirujanos y/o especialistas que realizaron su consulta externa durante el mes de ejecución y que aceptaron formar parte del estudio. Posteriormente se procedió a encuestar a los pacientes atendidos por los médicos culminada su consulta y/o a los padres de los niños atendidos en el servicio de Pediatría; fueron excluidos los pacientes con algún tipo de retraso mental y/o condición que afecte su lucidez, que se encontraban hemodinámicamente inestables o quienes completaran de manera errónea la encuesta. Asimismo solo se incluyó a los participaron que brindaron adecuadamente su consentimiento informado.

La población inicial de nuestro estudio estuvo conformada por 40 médicos cirujanos distribuidos en los servicios antes mencionados. Sin embargo, se contó con una tasa de rechazo del 25\% (10 médicos) por lo que tuvieron que ser retirados al igual que sus pacientes.

\section{TÉCNICAS E INSTRUMENTOS DE RECOLECCIÓN DE DATOS}

La presente investigación se ejecutó una vez aprobado por el Comité de Ética de la Facultad de Medicina Humana de la Universidad Nacional de Ucayali. Se solicitaron los permisos de la institución y posteriormente a la dirección de Hospital Regional de Pucallpa para la ejecución del estudio. Posteriormente se les invitó a los médicos cirujanos a formar parte de la investigación, procediendo a llenar los instrumentos de recolección de datos con quienes aceptaron; una vez hecho esto, se procedió a encuestar a los pacientes atendidos de su consulta externa por el periodo de 2 semanas; a cada participante se le informó sobre la finalidad del estudio para invitarlo a participar del mismo.

La información se obtuvo a través de una encuesta que constaba de 2 cuestionarios; la primera que realizaba el diagnóstico de SBO empleando el Maslach Burnout Inventory (MBI) dirigido a los médicos cirujanos y la segunda para medir la percepción de la relación médico - paciente empleando la escala PREMEPA dirigida a los pacientes atendidos por consultorio externo. 
El Maslach Burnout Inventory (MBI) es una escala validada al castellano ${ }^{14}$, con un análisis factorial (cuestionario y sus componentes) con un alfa de Cronbach mayor a $0,7^{15}$. Consta de 22 ítems que mide tres componentes principales: despersonalización (DP) correspondiente a los ítems 5, 10, 11, 15, 22, agotamiento emocional (AE) correspondiente a los ítems 1, 2, 3, 6, 8, 13, 14, 16, 20 y realización personal (RP) correspondiente a los ítems 4, 7, 9, 12, 17, 18, 19, 21. Cada ítem cuenta con siete opciones de respuesta en una escala tipo Likert en donde 0 es "nunca" hasta 6 que corresponde a "todos los días"16. Los puntajes obtenidos miden niveles de alteración que cuentan los componentes despersonalización indicando un DP leve (0-5 puntos); DP moderado (6-9 puntos) y DP severo (10-30 puntos), agotamiento emocional indicando un AE leve (0-18 puntos); AE moderado (19-26 puntos); AE severo (27-54 puntos); además de la realización personal con indicadores de RP bajo (033 puntos); RP medio (34-39 puntos); RP alto (40-48 puntos) ${ }^{16}$. Finalmente, se consideró a aquellos quienes cumplían criterios como un nivel severo de $A E$, un nivel severo de DP y/o un bajo nivel de $\mathrm{RP}^{17}$. Durante nuestro estudio, se usó como punto de corte a partir de la presencia de dos o más criterios.

La escala PREMEPA instrumento cuya consistencia interna mediante la prueba de Kücler-Richarson fue de 0.83 , y un índice de fiabilidad del $91.3 \%{ }^{18}$. Consta de 14 ítems, el ítem 1 que identifica si el paciente busco ayuda previamente, los ítems del 2 al 9 con opciones mediante una escala Likert cuyos valores van desde el 0 al 3 que evalúan las características que posee el profesional para con sus pacientes, los ítems del 10 al 13 poseen una escala dicotómica cuyos valores oscilan 0 puntos (respuesta negativa) y 3 puntos (respuesta afirmativa). La sumatoria de los puntajes evalúa tres categorías conformada por una adecuada relación médico paciente ( $\geq 15$ puntos); medianamente adecuada relación médico paciente (entre 14 a 9 puntos) y una inadecuada relación médico paciente ( $\leq$ 8 puntos). Así mismo, el ítem 14, evaluó la satisfacción del paciente con la atención médica, mediante una escala vigesimal otorgado encontrándose muy satisfechos (> a 15 puntos), poco satisfechos (< de 10 puntos) y medianamente satisfecho; con un puntaje intermedio ${ }^{18}$.

Adicionalmente, se designaron seis dimensiones, los cuales se encargaban de medir dimensiones de empatía empleados por los ítems 3 y 4 del cuestionario PREMEPA (valor de 0-6 puntos); la dimensión cortesía, ítem 2 (valor de 0-3 puntos); la dimensión humanidad, ítem 5 (valor de 0-3 puntos); la dimensión confianza, ítem 6 (valor de 0-3 puntos); la dimensión participación, ítems 7,8,10,11 (valor de 0 - 10 puntos); y para medir la dimensión intención de retorno, el ítem 12 del cuestionario (valor de $0-3$ puntos) ${ }^{19}$.

Además, se evaluaron las características generales del médico en el que se incluye el sexo, especialidad, horario de trabajo a la semana, años de experiencia. La última sección que evaluaba las características generales del paciente que incluye sexo, edad, tipo de paciente (nuevo, continuador), grado de instrucción, ingreso familiar mensual, ocupación y estado civil.

Una vez recolectada la muestra de los médicos cirujanos y pacientes atendidos en los consultorios externos se procedió a determinar quiénes cumplieron los criterios con SBO. Posteriormente, se obtuvo el puntaje de los niveles de relación médicopaciente y satisfacción de la consulta medidos desde la percepción del paciente, se calculó el promedio de los mismos para su análisis. Se procedió a analizar los datos con el paquete estadístico STATA 15.0, en el que se presentó en frecuencias y porcentajes las variables cualitativas; y para las variables cuantitativas, media y desviación estándar si presentaban una distribución normal. Se emplearon modelos lineales generalizados mediante la familia de Poisson para la estadística inferencial mediante razones de prevalencia crudos (RPc) y ajustados (RPa) según las características más importantes, considerándose un valor de $p<0,05$, como estadísticamente significativo.

\section{RESULTADOS}

La población de médicos estudiada estuvo conformada por 30 médicos cirujanos, siendo el 76,7\% (23) varones, la mediana de la edad fue de 44 años, con rangos intercuartílicos de 41-52. El 83,3 \% refirió que estaban casados y sólo el 13,4\% eran solteros. El $53.3 \%$ de los médicos procedían de la costa (Trujillo, Ica, Lima), el 26,7\% de la sierra (Junín, Arequipa, Cusco y Huánuco) y sólo el $20 \%$ en la selva (Ucayali).

Con respecto a su desempeño profesional se observó que el 33,3\% (10) de los médicos cirujanos no contaban actualmente con una especialidad, sólo el 6,7\% (2) de los médicos no contaban con un consultorio privado. Sólo el 46,7\% (14) de los participantes trabajaban menos de siete horas a la semana. La mediana de los años que ejercieron su carrera como médico cirujano fue de 18 años, con rangos intercuartílicos 9-27.

Dentro delos médicos especialistas, 16,7\% (5) eran ginecoobstetras; $10 \%$ (3) traumatólogos; 6,7\% (2) pediatras, 
neurólogos y médico internistas; se consideraron en otras especialidades como cardiología, infectología, anestesiología, cirugía general, urología y oftalmología.

Con respecto al SBO, la subescala de agotamiento emocional indicó que el 6,7\%; 86,6\% y 6,7\% contaban con un agotamiento emocional leve, moderado y severo, respectivamente. Los niveles de despersonalización indicaron que sólo el 3,3\% y el 96,7\% tenían un grado moderado y severo, respectivamente. En cuanto a la realización personal se determinó que el 76,7\% y; $23,3 \%$ tuvieron grados bajos y medios. Por lo que, se consideró en esta investigación a aquellos médicos con SBO quienes cumplían con dos de los tres de los componentes determinando que sólo el $20 \%$ (6) no padecían de agotamiento profesional (Tabla 1).

Tabla 1. Prevalencia de agotamiento profesional y sus características de sus componentes.

\section{N (\%)}

Agotamiento Emocional

Leve

$2(6,7 \%)$

Moderado

$26(86,6 \%)$

Severo

$2(6,7 \%)$

Despersonalización

Moderado

$1(3,3 \%)$

Severo

$29(96,7 \%)$

Realización Personal

Bajo

$23(76,7 \%)$

Medio

$7(23,3 \%)$

Síndrome de Burnout

Sin agotamiento profesional

$6(20 \%)$

Con agotamiento profesional

$24(80 \%)$

Con respecto a los pacientes atendidos en el consultorio externo, el 69,1\% (145) fueron mujeres. Sólo 48,6\% (102) estaban casados y el 35,2\% (74) contaban con ingreso mensual menor a los 900 nuevos soles, sólo mientras el 19,1\% (40) percibían un ingreso mayor de 1500 nuevos soles. Respecto al grado de institución $3,8 \%$ (8) no contaban con estudios, 5,2\% (11) sólo tenían estudios primarios y el $48,6 \%$ (102) contaba con estudios superiores.

Con respecto al grado de satisfacción de la consulta el $20 \%$ (42) manifestaron estar muy satisfechos, sólo el $1 \%$ (2) estuvieron poco satisfechos. Asimismo, el 83,3\%
(175) tuvieron una adecuada relación médico paciente y el 16,7\% (35) una mediana relación médico paciente. A pesar de ello se pudo evidenciar que no se contó con una baja o inadecuada relación médico paciente.

De acuerdo a la media en las dimensiones del cuestionario PREMEPA la Empatía, Cortesía, Humanidad, Confianza, Participación e Intención de Retorno se obtuvieron resultados cerca del puntaje máximo. Todas las dimensiones del cuestionario muestran un coeficiente de variación (Cv) inferior al $50 \%$, mostrando una distribución homogénea de los datos (Tabla 2). 
Tabla 2. Dimensiones de la percepción de la relación médico paciente desde la perspectiva de los pacientes del Hospital Regional de Pucallpa.

\begin{tabular}{lccccc|}
\hline & & & & \multicolumn{2}{c|}{$\begin{array}{c}\text { 95\% de confianza para la } \\
\text { diferencia }\end{array}$} \\
\hline Percepción & $\mathbf{X}$ & D.E. & C.V. & Inferior & Superior \\
\hline Empatía & 4.55 & 0.84 & 18.46 & 4.44 & 4.66 \\
\hline Cortesía & 2.80 & 0.40 & 14.29 & 2.75 & 2.85 \\
\hline Humanidad & 2.80 & 0.40 & 14.29 & 2.75 & 2.85 \\
\hline Confianza & 2.22 & 0.43 & 19.37 & 2.16 & 2.28 \\
\hline Participación & 7.46 & 0.85 & 11.39 & 7.34 & 7.58 \\
\hline Retorno & 2.90 & 0.54 & 18.62 & 2.83 & 2.97 \\
\hline
\end{tabular}

X: Promedio

D.E.: Desviación Estándar

C.V.: Coeficiente de variación

Con respecto al análisis crudo, no se encontró asociación inicial entre el SBO y la edad y el sexo por lo que no fue considerado dentro de nuestra investigación, sin embargo la residencia médica y el servicio médico si tuvieron relación estadísticamente significativa con el SBO. Con respecto al análisis ajustado, se pudo determinar que aquellos médicos que realizaron la residencia médica tenían $38 \%$ menos probabilidad de padecer de SBO (RPa: 0.62; IC 95\%: 0.62 - 0.77) en comparación de aquellos médicos que ejercían sin haber realizado la residencia, el encontrarse trabajando en los consultorios de cirugía predispuso a que tuvieran 1.46 (RPa: 1.46; IC 95\%: 1.20 1.76) más veces de presentar agotamiento profesional, al igual que aquellos que trabajaban en el servicio de ginecología, presentando $69 \%$ más probabilidades de padecer síndrome de Burnout (RPa: 1.69; IC 95\%: 1.41 2.03) en comparación de aquellos que se encontraban en el servicio de medicina (Tabla 3 ).

Tabla 3. Análisis crudo y ajustado del síndrome de burnout y sus factores asociados.

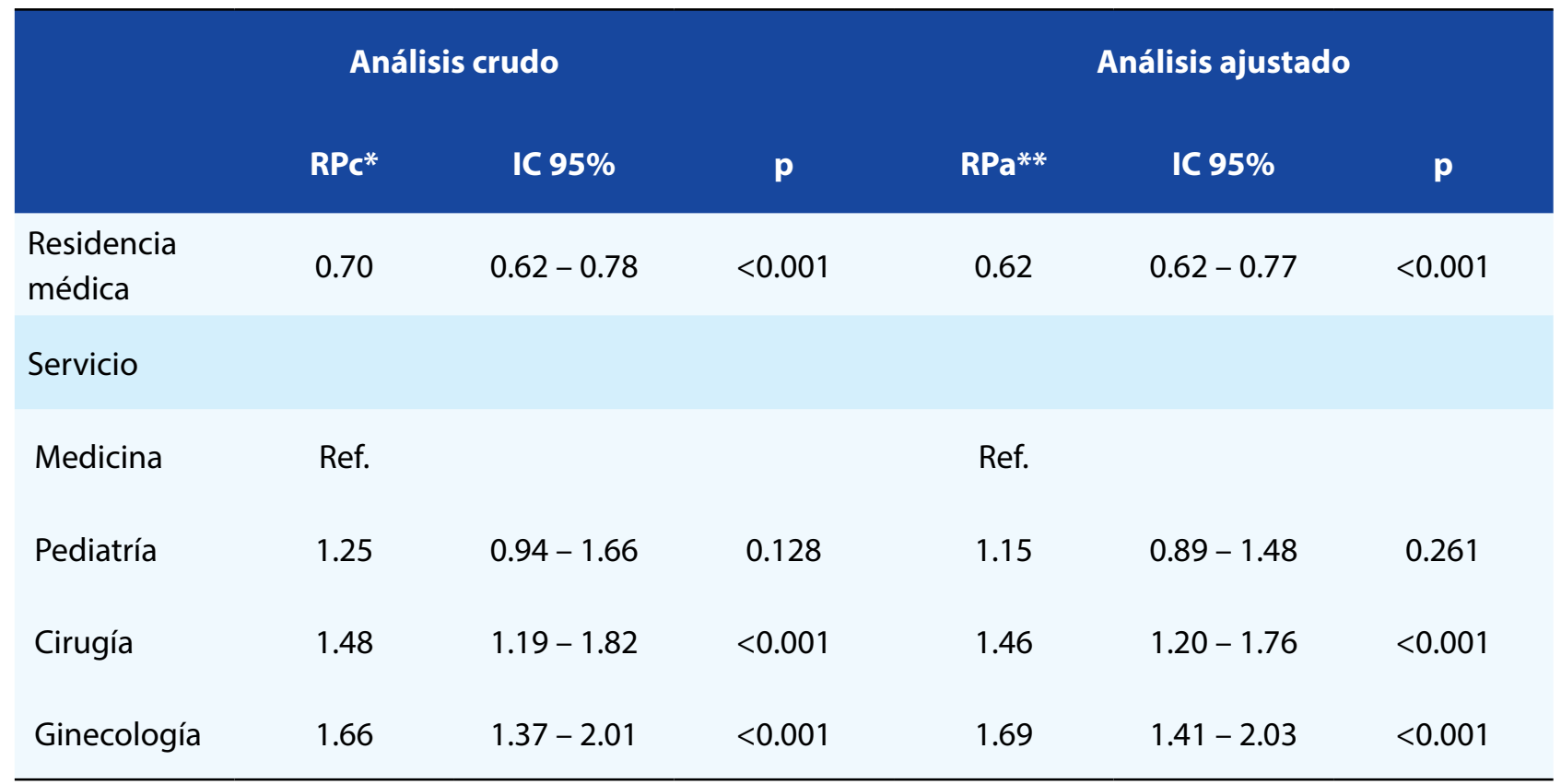


Finalmente, se encontró que los médicos que contaban con SBO tenían 59\% (RPa: 1.59; IC 95\%: 1.22 - 2.07) más probabilidades de realizar una inadecuada mala consulta y/o obtener poca satisfacción desde la perspectiva del paciente (Tabla 4).

Tabla 4. Síndrome de burnout y calidad en la atención hospitalaria en un hospital de la Amazonia peruana.

$\begin{array}{llllll}\text { Análisis crudo } & & \text { Análisis ajustado } \\ \text { RPc* } & \text { IC } 95 \% & \text { P } & \text { RPa* } & \text { IC } 95 \% & \text { P }\end{array}$

Relación médico - paciente

Relación médico-paciente mediana

Ref.

Ref.

\begin{tabular}{|c|c|c|c|c|c|c|}
\hline Relación médico-paciente adecuada & 0.87 & $0.70-1.09$ & 0.234 & 0.92 & $0.76-1.11$ & 0.398 \\
\hline \multicolumn{7}{|l|}{ Satisfacción de la consulta } \\
\hline Muy satisfecho & Ref. & & & Ref. & & \\
\hline Medianamente satisfecho & 1.10 & $0.91-1.33$ & 0.329 & 1.14 & $0.96-1.35$ & 0.125 \\
\hline Poco satisfecho & 1.35 & $1.13-1.62$ & 0.001 & 1.59 & $1.22-2.07$ & $<0.001$ \\
\hline
\end{tabular}

${ }^{*}$ RPc: Razón de prevalencia cruda

**RPa: Razón de prevalencia ajustada

\section{DISCUSIÓN}

En la actualidad, se han realizado múltiples investigaciones quienes delimitan la prevalencia de Burnout en diferentes poblaciones presente desde los estudiantes en el pregrado ${ }^{20}$, internos ${ }^{21}$, residentes ${ }^{22}$ y asistentes $^{23}$ de medicina humana. Ocho de cada diez médicos en nuestro estudio reportaron presentar SBO, frecuencias menores registradas en Tacna ${ }^{24}$, Colombia ${ }^{25}$, México ${ }^{26,27}$ y Argentina ${ }^{28}$ con frecuencias que oscilaron entre un 5\% - 45\%. Sin embargo, también se hallaron cifras similares al nuestro en México ${ }^{29}$.

Toda esta investigación nos refleja una alta tasa de agotamiento profesional en etapas muy tempranas, en la que el médico cirujano se encuentra sometido a diversas situaciones de estrés, no sólo desde su formación sino hasta su propio desempeño en el campo laboral. Se ha demostrado que el agotamiento profesional suele aumentarse debido a diversos eventos adversos en la prestación de servicios médicos, tanto en su deterioro del aprendizaje como en el incremento de los trastornos depresivos ${ }^{21}$.

Del mismo modo, las atenciones brindadas por los servicios de cirugía y gineco - obstetricia predisponía a los médicos en padecer agotamiento profesional, similar a lo encontrado en Brasil ${ }^{23}$ y México ${ }^{30}$ los cuales nos indican que las ramas quirúrgicas en nuestro país conllevan también a nuestros médicos a niveles altos de agotamiento emocional, despersonalización y/o posiblemente niveles bajos de realización personal. Es por ello, que se han realizado diversas intervenciones con la finalidad de reducir las horas de los médicos cirujanos con SBO, sin embargo, hasta el momento sólo han sido efectivas las intervenciones más no se ha podido reducir la incidencia de SBO en su totalidad ${ }^{31}$.

El realizar el residentado médico o contar con una especialidad representó hasta un 38\% menos probabilidad de padecer SBO, una realidad difícil de contrastar dado que se encontraron resultados contradictorios que reafirman que las especialidades médicas son quienes más lo llegan a padecer ${ }^{32-37}$. A pesar de ello, un estudio realizado en un hospital peruano identificó que sólo $27,8 \%$ de sus médicos especialistas había $\mathrm{SBO}^{38}$. Este factor protector podría deberse a los años de experiencia y/o gran número de horas de práctica hospitalaria que cuentan los médicos los cuales le proporcionan mayor confianza y un alto nivel de realización de personal (uno de los componentes de SBO). Además, el contar con un nivel de preparación superior explicaría el motivo de la confianza frente a sus colegas en la consulta externa, representando un factor protector para el desarrollo de SBO. 
Nuestro estudio encontró que los médicos que tienen SBO brindan una atención poco satisfactoria desde la percepción del paciente. Pocos estudios han sido descritos sobre la relación entre la consulta médica y el SBO. Algunos estudios sugieren que un aumento en los niveles de $\mathrm{SBO}$ en los médicos causa una relación médico paciente tensa lo que conlleva a una poca satisfacción en la consulta. Sin embargo, se ha visto que existen otras medidas como el mejorar los niveles de conocimientos o autoaprendizaje, la autoevaluación y los grados de SBO, el brindar un asesoramiento psicológico para proporcionar medios de alivio a los médicos, medidas de reducción para controlar la producción y el agravamiento del agotamiento laboral en doctores como medida crucial para mejorar la satisfacción del paciente en la consulta ${ }^{39,40}$.

La relación médico-paciente es la base de la práctica clínica médica. En las últimas décadas cambios como el desarrollo tecnológico y el mayor grado de especialización, han influenciado en esta relación, el grado de satisfacción de los médicos respecto a su práctica hospitalaria también han sido considerado como determinantes principales. La mayoría de los estudios realizados han prestado poca atención en cómo los médicos experimentan y enfrentan estos cambios en su profesión ${ }^{41}$. Nuestra investigación pudo identificar la percepción por parte de los pacientes con respecto a la relación médico paciente y cada uno de sus componentes; sin embargo, no se identificó la calidad de la relación médico-paciente desde la percepción del médico, por lo que se recomienda que esto sea estudiado de manera bidireccional en futuras investigaciones.

La investigación presentó ciertas limitaciones como la tasa de rechazo de los médicos cirujanos por participar y encuestar a sus pacientes posterior a la consulta médica, además de no realizar un estudio caso control y/o longitudinal para poder determinar su causalidad. Nuestra investigación, recomienda mejorar el sistema de salud, distribuyendo adecuadamente la cantidad de pacientes por médicos para aumentar el tiempo de consulta y poder mejorar percepción y satisfacción por parte del paciente de manera proporcional para poder realizar un acompañamiento psicológico a los médicos y determinar a un futuro su agotamiento profesional brindando consejería adecuada.

\section{CONCLUSIÓN}

Nuestra investigación puede concluir que ocho de cada diez médicos desarrolló SBO, los pacientes manifiestan tener niveles adecuados de relación médico-paciente a pesar del elevado índice de SBO, las especialidades quirúrgicas predisponen a los médicos a desarrollar SBO.

Contribuciones de autoría: Los autores participaron en la génesis de la idea, diseño de proyecto, recolección e interpretación de datos, análisis de resultados y preparación del manuscrito del presente trabajo de investigación.

\section{Financiamiento: Autofinanciado.}

Conflicto de interés: La presente investigación formó parte de la tesis de pre grado del médico cirujano Mario Milton Marchand Gonzales. Sin embargo, los autores de la presente investigación declaran no presentar ningún tipo de conflicto de interés.

Recibido: 22 de julio del 2019

Aprobado: 11 de setiembre del 2019

Correspondencia: Jennifer Steffany Vilchez Cornejo

Dirección: Jr. Ramón Castilla 159 Mz E Lote 11

Teléfono: 005161961506868

Correo:j.vilchezcornejo@gmail.com

\section{REFERENCIAS BIBLIOGRÁFICAS}

1. Freudenberger HJ. Staff burn-out. J Soc Issues. 1974;30(1):159-165 Disponible en: http://doi:10.1111/j.1540-4560.1974.tb00706

2. Kim MH, Mazenga AC, Simon K, Yu X, Ahmed S, Nyasulu P, et al. Burnout and self-reported suboptimal patient care amongst health care workers providing HIV care in Malawi. PLoS One. 2018;13(2):e0192983. Disponible en: https://doi.org/10.1371/journal.pone.0192983 .

3. Saijo Y, Chiba S, Yoshioka E, Kawanishi Y, Nakagi Y, Ito, T et al. Job stress and burnout among urban and rural hospital physicians in Japan. Aust $J$ Rural Health. 2013;21(4):225-231. Disponible en: https://doi.org/10.1111/ ajr.12040 .

4. Maslach C, Jackson SE, Leiter MP. Maslach Burnout Inventory Manual. 4th ed Menlo Park, CA: Mind Garden Inc; 2016.

5. Carlin M. El síndrome de burnout: Evolución histórica desde el contexto laboral al ámbito deportivo. Anales de psicología, 2010; 26(1), 169. Disponible en:https://www.um.es/analesps/v26/v26_1/20-26_1.pdf
6. Dávila FA, Nevado N. Validación de Burnout screening inventory en personal de formación del área de la salud. Educación Médica, 2016; 17(4) 158-63. Disponible en: http://dx.doi.org/10.1016/j.edumed.2016.08.007

7. Stavroula Leka. La organización del trabajo y el estrés. OMS. 2014 Disponible en:https://www.who.int/occupational_health/publications/ pwh3sp.pdf?ua=1

8. Rosende M, Kesque J, Romero MB, Rosende MC, Nelida-Severino M. Síndrome de Burnout: Padecimiento en instituciones públicas. 2009. Disponible en: http://psiqu.com/1-4129.

9. Arrogante Ó. Mediación de la resiliencia entre burnout y salud en el personal de Enfermería. Enfermería Clínica, 2014. 24(5): 283-9. Disponible en: https://doi.org/10.1016/j.enfcli.2014.06.003 .

10. Roldán, A. M. A., \&amp; Barriga, A. M. Q. Síndrome por quemarse en el trabajo y variables familiares y laborales de los médicos generales de Bogotá. Una estrategia de calidad laboral. Revista Colombiana de Psiquiatría, 44(4), 2015; 198-205. Disponible en: https://doi.org/10.1016/j. rcp.2015.05.017 
11. Devi S. Doctors in distress. Lancet. 2011;377:454-5. Disponible en https://doi.org/10.1016/S0140-6736(11)60145-1

12. Maticorena-Quevedo J, Beas R, Anduaga-Beramendi A, Mayta-Tristán P. Prevalencia del síndrome de burnout en médicos y enfermeras del Perú, Ensusalud 2014. Rev Peru Med Exp Salud Publica. 2016;33(2):241-7. Disponible en: http://dx.doi.org/10.17843/rpmesp.2016.332.2170

13. Kumar S. Burnout and Doctors: Prevalence, Prevention and Intervention. Healthcare 2016, 4(3), 37. Disponible en: https://doi. org/10.3390/healthcare4030037

14. Gil-Monte P. Validez factorial de la adaptación al español del Maslach Burnout Inventory General Survey. Salud Publica Mex. 2002; 44: 33-40. Disponible en: http://www.insp.mx/salud/index.html

15. Cáceres-Mejía B, Roca-Quicaño R, Torres M, Pavic-Espinoza I, Mezones Holguín E, Fiestas F. Análisis factorial del "Cuestionario para la evaluación del síndrome de quemarse por el trabajo" en estudiantes de medicina peruanos. Rev Psiquiatr Salud Ment (Barc.). 2013. Disponible en: http:// dx.doi.org/10.1016/j. rpsm.2013.06.002.

16. Nakandakari MD, De la Rosa DN, Failoc-Rojas VE, Huahuachampi KB Nieto WC, Gutiérrez Al, et al. Síndrome de burnout en estudiantes de medicina pertenecientes a sociedades científicas peruanas: Un estudio multicéntrico. Rev Neuropsiquiatr. 2015;78(4): 203-210. Disponible en http://www.scielo.org.pe/scielo.php?script=sci_arttext\&pid=S0034 $85972015000400003 \&$ Ing $=e s$

17. Vásquez-Manrique JF, Maruy-Saito A, Verne-Martín E. Frecuencia del síndrome de burnout y niveles de sus dimensiones en el persona de salud del Servicio de Emergencia de Pediatría del Hospital Nacional Cayetano Heredia en el año 2014. Lima, Perú. Rev Neuropsiquiatr. 2014 77 (3): 168-174. Disponible en: https://doi.org/10.20453/rnp.v77i3.2031

18. Ramos R. Percepción de las relaciones médico-paciente por parte de los usuarios externos del Departamento de Medicina del Hospital Carrión, Callao-Perú Lima; 2008. Disponible en: https://doi.org/10.15381/ anales.v69i1.1173

19. Núñez-Morales $R$, Arias-Cornejo $M$, Sánchez-Sánchez $N$, HernándezPorras T, Bustamante-Bravo V, Reupo-Vallejos M et al. Relación médicopaciente desde la perspectiva del paciente en el Hospital Naciona Almanzor Aguinaga Asenjo 2014. Rev. Exp. Med. 2015; 1 (1); 20-4 Disponible en: http://rem.hrlamb.gob.pe/index.php/REM/article/view/12

20. Vilchez-Cornejo J, Huamán-Gutiérrez R, Arce-Villalobos L, MoránMariños C, Mihashiro-Maguiña K, Melo-Mallma N et all. Síndrome de burnout en estudiantes de medicina: frecuencia, características y factores asociados. Act Med Per, 2016. 33(4):282-8. Disponible en: http://www. amp.cmp.org.pe/index.php/AMP/article/view/221/169

21. Reyes-Ticas A, Medina MT, Mesa X, Paredes Y, Barahona Y, Sierra M. Estudio de síndrome de Burnout, depresión y factores asociados en los practicantes internos del Hospital Escuela. Rev Fac Cienc Med. 2012; 9(1): 14-30. Disponible en: http://www.bvs.hn/RFCM/pdf/2012/pdf/ RFCMVol9-1-2012-4.pdf.

22. Miyoshi R, Matsuo $H$, Takeda R, Komatsu $H, A$ be $H$, Ishida Y. Burnout in Japanese residents and its associations with temperament and character Asian J Psy. 2016; 24: 5-9. Disponible en: https://doi.org/10.1016/j. ajp.2016.08.009

23. Nobre de Novais R, Rocha LM, Eloi RJ, Dos-Santos LM, Moura MV Ribeiro $\mathrm{R}$ et all. Burnout síndrome prevalence of on-call surgeons in a trauma reference hospital and its correlation with weekly workload cross-sectional study. Disponible en: https://doi.org/10.1590/0100 69912016005003

24. Dueñas $M$, Merma L. Prevalencia de Burnout en médicos de la ciudad de Tacna. CIMEL. 2003;8(1):34-8. Disponible en: http://www.redalyc.org/ articulo.oa?id=71780107

25. Paredes G, Olga L; Sanabria-Ferrand, Pablo A. Prevalencia del síndrome de burnout en residentes de especialidades médico quirúrgicas, su relación con el bienestar psicológico y con variables sociodemográficas y laborales. Revista Med. 2008; 16 (1): 25-32. Disponible en: http://www. redalyc.org/articulo.oa?id=91016105
26. Aguilera EC, de Alba García JEG. Prevalencia del síndrome de agotamiento profesional (burnout) en médicos familiares mexicanos: análisis de factores de riesgo. Rev Col Psiq. 2010; 39(1): 67-84. Disponible en: https://10.1016/S0034-7450(14)60237-7

27. Sánchez-Cruz J, Mugártegui-Sánchez S. Síndrome de agotamiento profesional en los médicos familiares. Rev Med Inst Mex Seguro Soc. 2013; 51(4): 428-31. Disponible en: http://revistamedica.imss.gob.mx/ editorial/index.php/revista_medica/article/viewFile/943/1530

28. Ponce LB, Gavotti GC, Ferreyra L, Flores M. Síndrome de Burnout en médicos residentes del Hospital Privado. Año 2013. Experiencia Médica. 2016; 33 (1): 08-11. Disponible en: https://experienciamedicahp.com.ar/ uploads/articulo-original-2-6136.pdf

29. Terrones-Rodríguez JF, Cisneros-Pérez V, Arreola-Rocha JJ. Síndrome de burnout en médicos residentes del Hospital General de Durango, México. Rev Med Inst Mex Seguro Soc. 2016; 54(2): 242-8. Disponible en: https://www.medigraphic.com/pdfs/imss/im-2016/im162p.pdf

30. Balcázar-Rincón LE, Montejo-Fraga LF, Ramírez-Alcántara YL. Prevalencia del síndrome de desgaste profesional en médicos residentes de un hospital de Mérida, Yucatán, Mexico. Atencion Fam. 2015; 22(4): 111-4. Disponible en: https://doi.org/10.1016/S1405-8871(16)30064-5

31. Gelfrand DV, Podnos YD, Carmichael JC, Saltzman DJ, Wilson SE, Williams RA. Effect of the80-hour workweek on resident Burnout. Arch Surg. 2004;139(9):933-8;discussion 938-40. Disponible en: https://doi. org/10.1001/archsurg.139.9.933

32. Kuan-Yu Ch, Che-Ming Y, Che-Hui L, Hung-Yi Ch, Mau-Roung L,Hui$\mathrm{Ru} C h$ et al. Burnout, Job Satisfaction, and Medical Malpracice among physicians. Int J Med Sci 2013;10(11):1471-1478. Disponible en: https:// dx.doi.org/10.7150\%2Fijms.6743

33. Grau A, Flichtentrei D, Suñer R, Prats M, Braga F. Influencia de factores personales, profesionales y transnacionales en el síndrome de Burnout en personal sanitario hispanoamericano y español (2007). Rev Esp Salud Pública 2009;83:215-30. Disponible en: http://scielo.isciii.es/pdf/resp/ v83n2/original1.pdf

34. Joffre-Velázquez VM, Peinado-Herreros JM, Gómez MC, Lin-Ochoa D, Vázquez-Nava F, Llanes-Castillo A. Síndrome de burnout en médicos de un hospital general en el noreste de México. Rev. Elec. Medicina, Salud y Sociedad. 2010; 1(1): 1-23. Disponible en: http://dx.doi.org/10.25009/ remsys.2010.1.18

35. Aranda-Beltrán C, Zárate-Montero B, Pando-Moreno M, SahúnFlores JE. Síndrome de Burnout, variable sociodemográficas del hospital Valentín Gómez Farias, México. Rev Col Sal Ocupacional 2011;1(1):1317. Disponible en: https://revistas.unilibre.edu.co/index.php/rc_salud_ ocupa/article/view/4794/4480

36. Castillo-Ávila I, Orozco CJ, Alvis ELR. Síndrome de burnout en el personal médico de una institución prestadora de servicios de salud de Cartagena de Indias. Rev Univ Ind Santander. Salud (Internet) 2015 [citado 4 setiembre 2019];47(2): 187-92.Disponible en: http://www.scielo.org.co/ scielo.php?script=sci_arttext\&pid=S0121-08072015000200010\&lng=en

37. Radman S, Gopal K. Prevalence and associated factors of burnout among doctors in Yemen. J Occup Health 2010;52:58-65. Disponible en: http://dx.doi.org/10.1539/joh.08030

38. Aldave Salazar MA. Síndrome de burnout y factores de riesgo en médicos Hospital Nacional Guillermo Almenara Irigoyen 2015. [Tesis doctoral].Lima: Universidad de San Martin de Porres, facultad de medicina humana; 2016. Disponible en: http://www.repositorioacademico.usmp. edu.pe/bitstream/usmp/2944/3/aldave_smr.pdf

39. Cohen D, Rhydderch M, Reading P, Williams S. Doctors' health: obstacles and enablers to returning to work. Occup Med (Lond) 2015; 65: 459-65. Disponible en: http://dx.doi.org/10.1093/occmed/kqv056

40. Merrett A, Jones D, Sein K, Green T, Macleod U. Attitudes of newly qualified doctors towards a career in general practice: a qualitative focus group study. Br J Gen Pract 2017; 67: e253-9. Disponible en: http://dx.doi. org/10.3399/bjgp17X690221

41. Zugar A. Dissatisfaction with medical practice. N engl J. Med 2004 350: 69- 75. Disponible en: http://dx.doi.org/10.1056/NEJMsr031703 